\title{
BREVE HISTORIA Y SENTIDO DE LA EXTENSIÓN UNIVERSITARIA
}

\author{
Patricio Donoso*
}

\section{RESUMEN}

La extensión universitaria, si bien constituye una de las actividades fundamentales del quehacer de la universidad, ha recibido muy poca atención si se la compara con otras áreas de este quehacer, como son la docencia y la investigación.

Esta falta de preocupación por el tema se ha traducido en una gran heterogeneidad de visiones y opiniones sobre el sentido, los alcances y la relevancia de esta tarea. Muchos confunden la extensión con la difusión. Otros tantos no distinguen entre extensión académica, educación continua o extensión artísticocultural. Algunos consideran la actividad de los servicios como extensión, otros no. Pocos son los que reconocen en las labores de extension, no sólo un instrumento y una plataforma para la proyección de la reflexión o la creación universitaria, sino también como un espacio y una instancia de contacto con las necesidades y los requerimientos de la sociedad.

Por esta razón, que la extensión universitaria es concebida por algunos como función de complemento, y por otros, como tarea primordial de la acción de una universidad. El objetivo del presente articulo apunta a entregar una "aproximación» al tema de la extensión universitaria, basada principalmente en una reseña de elementos históricos, y en un análisis a luz de la experiencia del autor sobre la materia.

* Docente, Pontificia Universidad Católica de Chile. 
HIGHER EDUCATION EXTRAMURAL ACTIVITIES IN BRIEF

\section{ABSTRACT}

Although extramural activities are one of the fundamental tasks carried out by universities, they have received very little attention compared to other university duties, such as research and teaching.

This lack of concern for the subject has translated into multiple different views and opinions on the meaning, the scope and the importance of extramural activities, which many people with dissemination. Others do not make a distinction between academic extramural activities, continuing education or cultural and artistic extension. Some people view activities carried out by university services as extramural activities while others do not. Only a few acknowledge extramural activities not only as a tool and a platform to project university thinking and creation but also as a space and a possibility for contact with the needs and requirements of society.

Therefore, extramural activities carried out by universities are viewed by some as a supplementary function while others consider it to be a primordial duty in the actions implemented by a university. The purpose of this article is to provide a first approach to the subject of extramural activities implemented by universities, based primarily on a review of historical elements and an analysis in light of the author's experience in this matter. 


\title{
BREVE HISTORIA Y SENTIDO DE LA EXTENSIÓN UNIVERSITARIA ${ }^{1}$
}

\author{
Patricio Donoso
}

\section{Tipología de la extensión universitaria}

En el entorno de la educación superior, se habla de la extensión: extensión académica, extensión artístico-cultural y extensión de la forma de servicios.

Por extensión artístico-cultural se entiende la acción de presentar o representar a un determinado público una creación en el ámbito artístico-cultural, como puede ser un concierto, una exposición de plástica, una obra teatral, un ciclo de cine, una conferencia en temas de cultura general, $u$ otras formas similares. Lo que se extiende en este caso, no es necesariamente el fruto de un trabajo de investigación o reflexión académica previa, sino la interpretación de la creación de un autor, o la exhibición del trabajo de éste.

Por extensión académica, se entiende: a) la acción de difundir y proyectar el resultado de la reflexión o la investigación académica más innovadora; b) la acción de entregar al público la oportunidad de actualizar o reciclar sus conocimientos, que también se denomina educación permanente o continuada; y c) la acción de crear o participar en espacios de interacción con públicos específicos o generales, en materias de valor académico.

Por extensión de la forma de los servicios se entienden los trabajos de asesoría o consultoria; las investigaciones o controles repetitivos; las atenciones médicas o psicológicas en hospitales o consultorios; las publicaciones de una editorial; los servicios de comunicación y

1. Este artículo se ha extractado del libro: Extension universitaria: Una aproximación para su análisis, del mismo autor, publicado por la Corporación de Promoción Universitaria (CPU), en 1993. 
promoción a través de medios de comunicación propios (radio, televisión, revistas); la interrelación con los ex-alumnos y amigos de la universidad, etc.

Algunas actividades pueden interpretarse como combinaciones de formas de extensión como las antes enunciadas: la capacitación y la educación a distancia, que pueden considerarse tanto de extensión académica como de extensión de la forma de servicios; un ciclo de cine acompañado de un foro-debate, que puede entenderse como extensión artístico-cultural y extensión académica.

Se entiende que la extensión no sólo consiste en extender el fruto o el producto del quehacer universitario; también supone extender su capacidad reflexiva y creativa, su carácter plural y universal, su visión multifacética y multidisciplinaria, etc.

Se entiende, por lo mismo, que la extensión es un proceso esencialmente comunicativo, en el que se produce una interacción centrífuga y centrípeta entre la universidad y la sociedad. También se entiende que la extensión es un servicio: servicio a la cultura, servicio a las personas, servicio a las instituciones, y servicio a la comunidad. En definitiva, la extensión universitaria es difusión, comunicación y servicio.

\section{Breve historia de la extensión universitaria en Chile}

\section{Los comienzos}

Las primeras actividades de este tipo ocurridas en Chile, que podrían denominarse de extensión universitaria, se vinculan con el requerimiento de difusión del quehacer de la universidad, y se remontan a la fundación o inicio de las actividades de algunas de las mismas. En la Universidad de Chile, por ejemplo, comienzan a publicarse los Anales de la Universidad en el año 1842, es decir, en el mismo año de su fundación, con el objeto de estimular la labor creativa y dar publicidad a los trabajos realizados por sus profesores ${ }^{3}$.

2. Krebs, Ricardo, "La extensión universitaria a través de la historia". Cuadernos del Consejo de Rectores de las Universidades Chilenas $\mathrm{V}^{\circ} 20$, mayo - agosto de 1983, págs. 6-7. 
Otro tanto ocurre con la Universidad Católica, a contar de su fundación en 1888, y con otras universidades creadas en forma posterior.

Durante las últimas décadas del siglo XIX y las primeras del siglo $\mathrm{XX}$, la actividad universitaria está marcada por la docencia profesional y la finalidad más decidida de las universidades es la formación de profesionales.

La extensión universitaria adquiere un rol singular en este contexto. El rectorado de Gregorio Amunátegui, en la Universidad de Chile, se caracteriza por la importancia dada a la extensión universitaria. El 17 de abril de 1923 se realiza en Chile la Quinta Conferencia Panamericana de Extensión Universitaria, que cuenta con la participación de destacados conferencistas extranjeros y chilenos ${ }^{3}$. En la Memoria anual de la Universidad Católica, de 1923, se definen objetivos relacionados con la extensión en la universidad: "La universidad, además de sus otros fines, debe desempeñar ella misma y directamente una obra de investigación e irradiación científica y cultural y de colaboración dentro de sus fines y medios propios, al bien público de su país y al bien general de la humanidad ${ }^{4}$.

La actividad de extensión se adecúa en esta época a los desarrollos observados en la acción universitaria, y tiene un fuerte acento en el potenciamiento de la labores prácticas y profesionales.

Un ejemplo claro en este sentido, y quizás una de las primeras definiciones estructuradas y más precisas de la función de la extensión en la universidad, es la que aporta el Decreto Ley $\mathrm{N}^{\circ} 4.807$ de 1929, que aprueba el Estatuto Orgánico de la Enseñanza Universitaria ${ }^{5}$.

En el artículo 53 de dicho Decreto, se define el rol de la universidad en el campo de la extensión, y se lo relaciona con lo que

3. Cuadernos de la Universidad de Chile Nol. "Medio siglo de vida universitaria: 1900-1950", Domingo Santa Cruz, pág. 20.

4. Krebs, Ricardo, Historia de la Pontificia Universidad Catolica de Chile. (Por publicarse).

5. Decreto Ley $\mathrm{N}^{\circ} 4.807$, del 4 de noviembre de 1929 , durante la presidencia de don Carlos Ibáñez del Campo, publicado en el Diario oficial, 18 de noviembre de 1929. 
hoy llamamos extensión académica y educación permanente o continuada. El artículo referido señala textualmente que: "Además de la función docente, la universidad propenderá al constante perfeccionamiento de su enseñanza y de la cultura general de la Nación, estableciendo para ello, trabajos de extensión universitaria por medio de los cursos que a continuación se indican: a) cursos libres; b) cursos de post-graduados; c) conferencias dentro y fuera de la universidad, y d) seminarios, trabajos de investigación científica y publicaciones".

El Decreto Ley $N^{\circ} 4.807$ establece un primer marco general para la actividad de extensión universitaria, centrado en forma especial en la educación permanente o continuada y en el fortalecimiento de una capacitación práctica de los alumnos, el que con el correr del siglo XX se va ampliando hacia otros fines $\mathbf{u}$ objetivos.

\section{Mitad de siglo XX: el auge de lo cultural}

Desde fines de la década del 1930 y principios de los años cuarenta, comienza a producirse un desarrollo casi vertiginoso en el campo de lo que podría denominarse extensión artístico-cultural.

El Departamento de Extensión de la Universidad Católica, por ejemplo, reorienta y refuerza sus actividades a una labor cultural artística. Según Krebs, "En 1941, el Rector señalaba que el Departamento consagraba sus esfuerzos a formar un movimiento capaz de irradiar y difundir un verdadero arte universitario, que tomara el lugar que le correspondía en la vida artística del pais ${ }^{6}$.

El teatro universitario surge en gloria y majestad. El 22 de junio de 1941, nace el pionero Teatro Experimental de la Universidad de Chile. Dos años más tarde, en octubre de 1943, surge el Teatro de Ensayo de la Universidad Católica. En 1945, inician sus actividades el TUC de la Universidad de Concepción y el TEKNOS de la Universidad Técnica del Estado.

Las autoridades universitarias toman clara conciencia de la importancia de estos desarrollos.

6. Krebs, Ricardo, Historia de la Pontificia Universidad Católica de Chile, op. cit. 
En su primer rectorado (1933-1938), Juvenal Hernández impulsa fuertemente la acción en el campo de la extensión universitaria. Del Departamento de Extensión Cultural, que siguió realizando conferencias y veladas cinematográficas, se origina una nueva actividad de gran vuelo: las llamadas Escuelas de Temporada. En la rama de las Artes Plásticas, se organizan salones oficiales. En el campo de la extensión musical, la Facultad de Bellas Artes, por intermedio del Conservatorio, comienza sus conciertos sinfónicos... ${ }^{7}$.

Durante las décadas de 1940 y 1950 se produce un gran desarrollo de todo tipo de expresiones de extensión cultural. Además del aporte en el campo del teatro universitario, en la Universidad de Chile se crean la Facultad de Ciencias y Artes Musicales y la Facultad de Ciencias y Artes Plásticas. Conjuntamente con ellas, se desarrollan diversas entidades dedicadas a la actividad de extensión: el Ballet Nacional Chileno, la Orquesta Sinfónica de Chile, el Coro Sinfónico y el de Cámara. En el campo de las artes plásticas, surgen con posterioridad el Museo de Arte Contemporáneo y el Museo de Arte Popular Americano. En la Universidad Católica, además del Teatro de Ensayo, se crean diversos conjuntos musicales a partir del Instituto de Música, como por ejemplo, el Coro de la Universidad. La Escuela de Arte, por su parte, además de formar a un número importante de destacados artistas plásticos nacionales, también realiza actividades de extensión y difusión en la forma de exposiciones y exhibiciones.

Otras universidades también realizan importantes aportes. La Universidad de Concepción edita la Revista Atenea y la Universidad Católica de Valparaíso convoca a las Primeras Jornadas Culturales.

3. Los años sesenta y principios de los años setenta: extensión y política

El concepto de extensión se amplia cada vez más. Ya no sólo está relacionado con la formación permanente y el reciclaje de los cuadros profesionales que se proponía a fines de la década de 1920, ni con las expresiones artístico culturales planteadas como servicio a la cultura y la sociedad de los cuarenta y cincuenta. La extensión universitaria

7. Cuadernos de la Universidad de Chile, op. cit., pág. 39. 
se transforma para algunos en un medio de influencia e intervención de la universidad en la sociedad. El rol de la extensión tiende a trascender los límites de la acción académica y comienza a sumergirse en el campo de lo político-contingente.

Hugo Montes explicita este nuevo rol, al señalar que: "La Universidad no puede ser elemento dirigido cuando se trata de cuestiones culturales; debe, por el contrario, dirigir a la sociedad, influir en ella, orientarla ${ }^{8} "$.

En el transcurso de la década de 1960 esta orientación e influencia comienza a extremarse, y la extensión pasa a ser un instrumento que supera el quehacer propiamente universitario. Comienzan a hacerse frecuentes menciones a conceptos como "conciencia crítica", "comunicación dialéctica", "procesos revolucionarios y de emancipación", "transformación social", "dominación-explotaciónmarginación", etc.

Como señala Ricardo Krebs, "En una Universidad comprometida con el pueblo, la extensión debía estar orientada hacia el proletariado urbano y campesino, debía contribuir a la transformación revolucionaria de las estructuras económicas, sociales y políticas y debía ayudar a que los hombres se librasen de toda forma de dependencia y alienación"".

\section{La extensión academizada}

Los cambios que experimenta Latinoamérica a partir de la década de 1970 , se trasmiten a la organización y operatoria de la actividad de extensión universitaria.

En la Universidad Católica, el nuevo Rector, almirante (R) Jorge Swett Madge, replantea el objetivo de la extensión universitaria, orientándola hacia una finalidad más académica y profesional. Plantea el Rector Swett que: "En una época en que el conocimiento científico se renueva en forma cada vez más acelerada, la política de extensión y

8. Montes, Hugo, "Humanismo y universidad", Revista Finis Terrae N45, 1964, pág. 14.

9. Krebs, Ricardo, La extensión universitaria a través de la historia, op. cit., pág. 9. 
comunicación debe tener por objetivo principal trasmitir el saber que se genera y renueva al interior de la universidad a las personas que han ingresado al mundo del trabajo. Extensión, debe ser, fundamentalmente, perfeccionamiento, capacitación y especialización. La universidad, centro del saber superior y en un proceso de continua reelaboración del saber, debe poner los adelantos del conocimiento científico y tecnológico a disposición de aquellos que aplican estos adelantos al desarrollo material y cultural de la nación ${ }^{10 "}$.

El nuevo rol de la extensión universitaria se recoge en el marco de la nueva política académica y administrativa aprobada por el Consejo Superior de la Universidad en 1978, en que se señala que: "La extensión es responsabilidad de las Facultades y de sus académicos, puesto que lo que se "extiende" es su trabajo previo, debiendo radicar en ellos su desarrollo. Cuando sea necesaria una acción coordinadora de varias unidades académicas, o cuando la naturaleza o magnitud de la actividad lo aconseje, le corresponderá intervenir, con dicho propósito, a la administración central ${ }^{11 "}$.

A principios de la década de 1980 la discusión sobre la función, objeto y sentido de la extensión comienza a hacerse nuevamente común en los foros universitarios.

El profesor Jorge Millas, en relación con la relevancia del quehacer de extensión plantea: "Ya es un poco excesivo considerar que la extensión sea una función esencial. Hay en ello un error semántico. Mucho menos lo es la pretendida esencialidad de la administración, pero ese error ya no es semántico, es de lógica. El semántico, relativo a la extensión, es más excusable. No, sin la extensión, la Universidad no perecería, porque el conocimiento continuaría prosperando en ella, inserto como formación espiritual y profesional en los jóvenes, y como nuevo conocimiento en la ciencia misma. No es pues, realmente esencial, la extensión para la Universidad. Pero es, en cambio, cosa necesaria y provechosa para la sociedad, y útil para la propia institución. Por ello, decir que, en rigor, la extensión es una función de

10. Krebs, Ricardo. Historia de la Universidad Católica de Chile, op. cit.

11. Documento politica académica y administrativa de la Pontificia Universidad Católica de Chile. Ediciones Universidad Católica de Chile, 1982, págs. 16-17. 
complemento y no de esencia, no es querer acabar con la extensión universitaria, sino poner orden en los conceptos y con ello orden, en las $\operatorname{cosas}^{12 "}$.

\section{El auge de los años noventa}

A fines de la década de 1980 y comienzos de los años noventa, varias universidades se replantean la necesidad de reforzar su trabajo en el campo de la extensión, lo cual da origen a las más diversas iniciativas de educación continuada o permanente. Se crean Programas de Extensión, Centros de Eventos, Centros Culturales, Centros de Extensión, y otras instancias similares. Las unidades del ámbito artístico-cultural incrementan en forma notable el número y calidad de sus eventos, y las unidades académicas realizan en forma sostenida y creciente actividades de extensión en sus respectivos campos, en la forma de cursos, seminarios, congresos, workshops, etc.

La discusión conceptual sobre la función y el sentido de la extensión universitaria es reemplazada por una visión más bien realista y pragmática del quehacer en este campo. Se busca, por sobre todo, atender a requerimientos y demandas concretas de la sociedad.

Con motivo de la creación del Centro de Extensión de la Pontificia Universidad Católica de Chile, el Rector Juan de Dios Vial plantea los nuevos desafíos de la extensión universitaria:

"La extensión debe responder a la necesidad cada vez más apremiante de asegurar a profesionales de las más diversas disciplinas un ambiente adecuado, a darles capacitación de alto nivel, a ponerlos al día en sus especialidades, a proporcionarles acceso al perfeccionamiento cultural, bajo el signo de la educación continuada, que está destinado a marcar, de modo cada vez más definido, el futuro de la vida profesional. Estamos conscientes de que la formación que le damos a un estudiante en nuestras aulas le sería suficiente para su vida profesional por un tiempo relativamente corto. Es ya normal que un profesional debe rehacer su formación varias veces en el curso de

12. Millas, Jorge. Misión de la universidad frente a las ciencias y a las artes. Idea y defensa de la universidad, 1981, pág. 111. 
su vida activa. La universidad no puede permanecer cerrada a ese signo de la época que es la necesidad de un permanente reciclaje de los estudios superiores. El Centro cobijará una actividad académica de las Facultades, cual es la educación continuada y la capacitación de alto nivel, y servirá, al mismo tiempo, de soporte para actividades hoy dispares en la universidad ${ }^{13 "}$.

\section{Sobre el futuro de la extensión}

La extensión universitaria, si bien parece tener un espacio ganado como función relevante dentro del quehacer universitario, no siempre es considerada al mismo nivel de importancia que las funciones tradicionales de docencia e investigación.

Ahora bien, si una universidad está preocupada en forma especial de vincularse con los problemas y situaciones concretas de su entorno, y pretende además responder prontamente a algunas de esas inquietudes externas, puede que la función de extensión deba tener en esa universidad un rol tan relevante como la docencia o la investigación.

Si la universidad está constituida por una serie de unidades que requieren en forma especial la interacción con el público externo, como parte de su proceso formativo y de desarrollo (por ejemplo: las escuelas de Teatro o Música), la extensión cumple un rol que va más allá de la investigación o la función docente.

La misión particular de una universidad o de una unidad de la misma es la que va a permitir definir el grado de esencialidad o complementariedad de la extensión universitaria.

Siguiendo el desarrollo histórico y el rol que en síntesis podría atribuirse al campo de la extensión universitaria, es posible afirmar que la extensión universitaria, en la medida que significa la proyección del mundo externo con la retroalimentación con del mismo, también es una función de primera importancia en el quehacer de la universidad.

13. Pontificia Universidad Católica de Chile, Cuenta de la Rectoría 1988/1989. 
Como señala José Ortega y Gasset, en su libro Misión de la Universidad, "No sólo necesita la universidad contacto permanente con la ciencia, so pena de anquilosarse. Necesita también contacto con la existencia pública, con la realidad histórica, con el presente, que es siempre un integrum y sólo se puede tomar en totalidad, sin amputaciones ad usum delphinis. La universidad tiene que estar también abierta a la plena actualidad; más aún, tiene que estar en medio dè ella, sumergida en ella. Y no digo esto sólo porque la excitación animadora del aire libre histórico convenga a la universidad, sino también, viceversa, porque la vida pública necesita urgentemente la intervención en ella de la universidad como tall"'. 